\title{
New Development of Remote Control System for Air Vehicle using 3G Cellular Network
}

\author{
Hiroshi Yamamoto Takuya Fujii Phuong Tran Thi Ha Katsuyuki Yamazaki \\ Department of Electrical Engineering, Nagaoka University of Technology \\ 1603-1 Kamitomioka, Nagaoka, Niigata 940-2188 Japan \\ Email: hiro_yama@ieee.org
}

\begin{abstract}
For obtaining various observation data in a dangerous disaster area where human beings cannot easily enter, a Delay Tolerant Network (DTN) based sensor network system using autonomous air vehicles has been developed. However, the existing system cannot help to observe disaster conditions of congested areas where sensor nodes cannot be easily deployed, although it can be used to collect sensor data from the sensor nodes deployed on a vast field. Therefore, in this study, we propose and develop a new air vehicle which can be controlled through a $3 \mathrm{G}$ cellular network so that an operator can obtain various sensor data in the disaster area from everywhere. Furthermore, the proposed air vehicle can be equipped with sensing devices because it has many analog/digital interfaces. Through the experimental evaluation, it is clarified that the remote control of the air vehicle can be done even when both the vehicle and the user terminal exist inside NAT devices.
\end{abstract}

Index Terms-Air Vehicle, Remote Control System, 3G Cellular Network, Sensor Network, DTN

\section{INTRODUCTION}

In a disaster observation field where human beings cannot easily enter and move around, a sensor network system has been attracting attention to automatically monitor disaster conditions [1], [2], [3], [4]. The existing sensor network system using wireless network (e.g., wireless LAN, ZigBee) is difficult to be used because sensing devices and network equipment cannot be deployed on the dangerous field, and special landscape or collapsed buildings block radio waves. A Delay Tolerant Network (DTN) based sensor network system using autonomous air vehicles has been proposed in order to solve a part of the problem [11]. In the proposed system, many sensor nodes are deployed in the disaster area, and the air vehicle autonomously travels about them to collect sensor data and carries it to a main server. However, the use of the air vehicle cannot help to observe disaster conditions of congested areas where the sensor nodes cannot be easily deployed, although a coverage area of the sensor network can be expanded to whole disaster area by using the air vehicle.

On the other hand, recently developed air vehicles (e.g., AR Drone [12], DJI Phantom [13]) are equipped with a camera device which can monitor detailed disaster conditions. In addition, many kinds of sensors and embedded systems (e.g., Arduino [14]) can be attached to them through serial or network interface, hence the air vehicle can also work as a sensor node that collects various sensor data everywhere even in the congested area. Furthermore, a $3 \mathrm{G}$ cellular network module for the embedded system has been developed so that the monitoring functions of the embedded system can be accessed through the Internet.

Therefore, in this study, we propose and develop a new air vehicle which can supports various monitoring functions and can be controlled from a location remote. This study proposes to use an AR Drone which is a quad-rotor air vehicle [12], and the monitoring function and remote control are achieved by attaching a embedded system with $3 \mathrm{G}$ cellular network module to the air vehicle. Through the experimental evaluation, it is concluded that the proposed air vehicle can capture conditions of the observation field and can be controlled through the $3 \mathrm{G}$ cellular network.

\section{RELATED WORKS}

\section{A. Sensor Network for Disaster Observation}

In resent years, a sensor network system is considered as an effective tool for disaster response, and has been widely studied by many researchers. In the disaster area, the sensor network should deliver high volumes of observation data in real time so that an operator can quickly make decisions. In addition, the sensor network should persistently work even when some sensor nodes fail due to accidents in the disaster area. In order to satisfy these requirements, several sensor network technologies have already been proposed.

First, a DistressNet is an ad hoc wireless architecture for disaster response, and supports distributed collaborative sensing, topology-aware routing using a multichannel protocol, and accurate resource localization [1]. Due to these features, accurate observation data (e.g., audio sensing) can be obtained from multiple sensors, and can efficiently be forwarded to the data sink by avoiding congested environments. Furthermore, for keeping persistence of the sensor data in the sensor network, a combination of a new network coding and replication technique that is referred to as a Growth Code, has been proposed [2]. On the other hand, in order to improve quality of medial activities in the disaster area, sensor nodes accommodating various types of sensors (e.g., vital, location sensors) have been developed [3], [4].

The existing sensor network system utilizes wireless communication technology (e.g., wireless LAN, ZigBee) for data communication between fixed sensor nodes. However, the wireless network is difficult to be used in the congested area 
of the observation field because special landscape or collapsed buildings block radio waves.

\section{B. Delay Tolerant Network}

Delay Tolerant Network (DTN) is currently focused as a solution for communication in heterogeneous or discontinuous network such as interplanetary Internet [5], [6]. The DTN is a general term which represents a set of technology required for achieving data forwarding in the network where communication links between nodes can be established only in a limited time interval. The key services of the DTN include in-network data storage, data transmission/retransmission method, naming method, and so on.

One of the popular data transmission methods of DTN is a store-carry-forward method, where a mobile node (e.g., satellite, car, truck) is used as a data ferry to collect various data from multiple data sources, store and carry the data back to the main server [7], [8]. The store-carry-forward method has been utilized for collecting various observation data (e.g., agricultural data, traffic data) [9], [10]. In the existing studies, a car or a truck carrying a DTN device is used as a data ferry, which moves on a field to collect data under human control.

However, in some observation fields where traditional road transportation is collapsed, it is difficult to apply these existing systems to the disaster response.

\section{DTN-based Sensor Network using Air Vehicle}

To solve the problems mentioned in Section II-B, an automatic system for gathering observation data in a DTN environment has been proposed [11]. Furthermore, the system should solve the problem of collecting data in some special field that a traditional transportation can not move easily. Therefore, in our previous study, an autonomous air vehicle has been proposed to be used as a data ferry for the DTNbased sensor network system [11]. This system can be used for disaster observation in a area where many buildings are collapsed, and the ferry automatically flies on the observation field to collect the data and then carries them back to the main server.

The use of the air vehicle can expand coverage area of the sensor network to whole disaster area, but cannot help to observe disaster conditions of congested areas where the sensor nodes cannot be deployed. Therefore, in this study, we propose a new air vehicle which can work as a sensor node supporting various monitoring functions and can be controlled from everywhere through $3 \mathrm{G}$ cellular network.

\section{Proposed Remote Control System for Air VEHICLE}

Figure 1 shows an overview of our proposed sensor network system using an autonomous air vehicle. As explained in this figure, specific features of our proposed air vehicle system are 1) autonomous flight algorithm for automated retrieval of sensor data (See Section II-C), and 2) remote control system for detailed disaster observation. In this section, we explain system structure of the proposed remote control system.

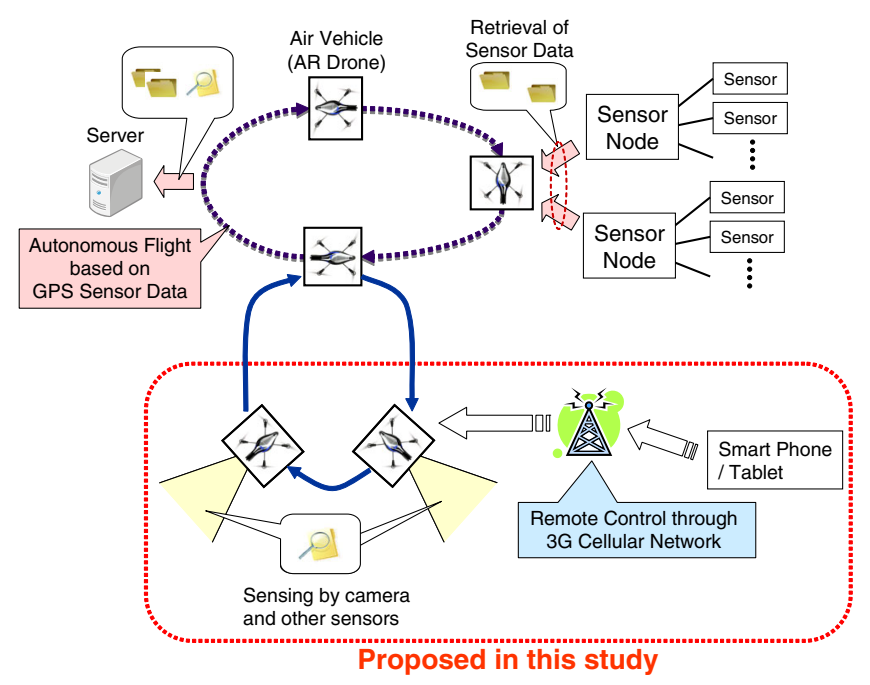

Fig. 1. Overview of Proposed Sensor Network.

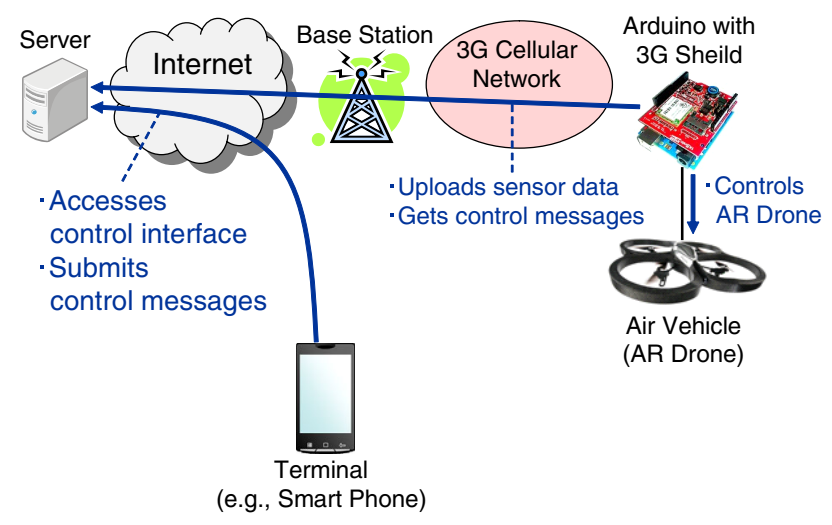

Fig. 2. Remote Control through Cellular Network.

\section{A. Overall Structure}

Figure 2 shows overall structure of our proposed remote control system using an air vehicle. As shown in this figure, the air vehicle is equipped with a network interface for $3 \mathrm{G}$ cellular network. Through the network interface, the air vehicle receives control messages (e.g., go forward, turn left) from a user terminal (e.g., smart phone, tablet) of an operator, and uploads various observation data (e.g., GPS data, sensor data, and video image) obtained from its equipment.

In the remote control system, a server is prepared for publishing a homepage which provides a control interface and which shows geographical location of the air vehicle. The server should be deployed on the Internet so that both the user terminal and the air vehicle can be operated inside NAT (Network Address Translation) devices. In the future, the homepage will display output of various sensors and video images taken by a camera device attached to the air vehicle.

When the operator controls the air vehicle in the observation field, the user terminal accesses the server to browse a homepage of the remote control interface. Through the control interface displayed on the browser, the operator can upload 
TABLE I

SPECIFICATION OF AR DRONE.

\begin{tabular}{|c|c|}
\hline Weight & $380[\mathrm{~g}](420[\mathrm{~g}]$ with indoor hull) \\
\hline Speed & $5[\mathrm{~m} / \mathrm{s}]$ \\
\hline CPU & ARM9 486 [MHz] \\
\hline Memory & $128[\mathrm{MB}]$ \\
\hline OS & Linux (modified version of Linux Kernel 2.6.27) \\
\hline Connection & Wifi (b/g), USB \\
\hline Sensor & MEMS 3-axis accelerometer, \\
& 2-axis gyro, \\
& Single-axis yaw precision gyrometer, \\
& Ultrasonic altimeter (range 6[m]), \\
& Wide-angle front camera, \\
& High speed vertical camera \\
\hline
\end{tabular}

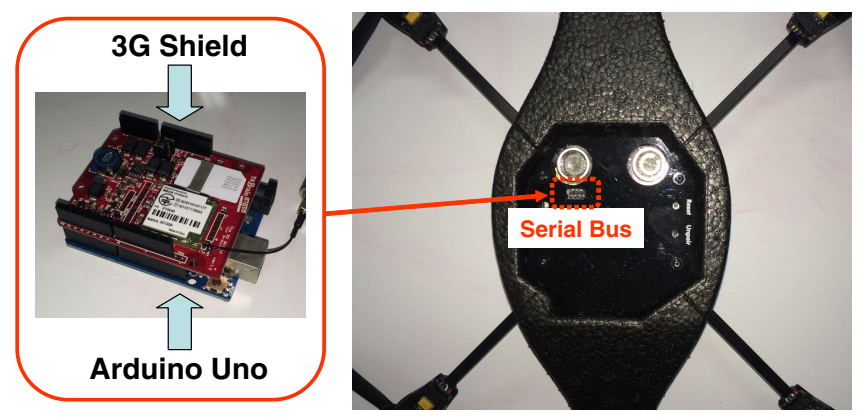

Fig. 3. Installation of 3G Shield on Air Vehicle.

control messages to the server. On the other hand, the air vehicle periodically obtains observation data from its GPS sensor and other sensors. When these data are uploaded to the server, the control messages are downloaded to the air vehicle through the cellular network. Finally, the flight of the air vehicle is controlled according to the downloaded messages.

\section{B. Implementation of Proposed Remote Control System}

In the proposed system, an AR Drone, a low-priced quadrotor helicopter developed by Parrot SA., is used as an air vehicle [12]. Detailed specification of the AR Drone is described in Tab. I. The AR Drone is equipped with several sensors (e.g., accelerometer, magneto-metric sensor, gyroscope) that help a stable flight of it, and has two types of interfaces (i.e., IEEE802.11b/g and Serial) for connecting to other devices. In addition, a front camera of the AR Drone can be utilized to take a picture of disaster conditions, and the picture can be stored on a data storage of the vehicle or can be uploaded to the Internet through the network interface. Furthermore, a Linux operating system is running on the AR Drone, hence we can easily implement necessary functions for the remote control as a Linux application.

As a network interface of the $3 \mathrm{G}$ cellular network, a single board micro-controller, Arduino Uno, with a $3 \mathrm{G}$ shield developed by TABrain Inc. [15] is utilized, and is attached to the AR Drone through a serial bus (TTL level) as shown in Fig. 3. The Arduino supports analog, digital, and serial interfaces, and hence helps the air vehicle to have extensibility of various sensors for disaster observation. Furthermore, the AR Drone can obtain its location information from a GPS function of the $3 \mathrm{G}$ shield.

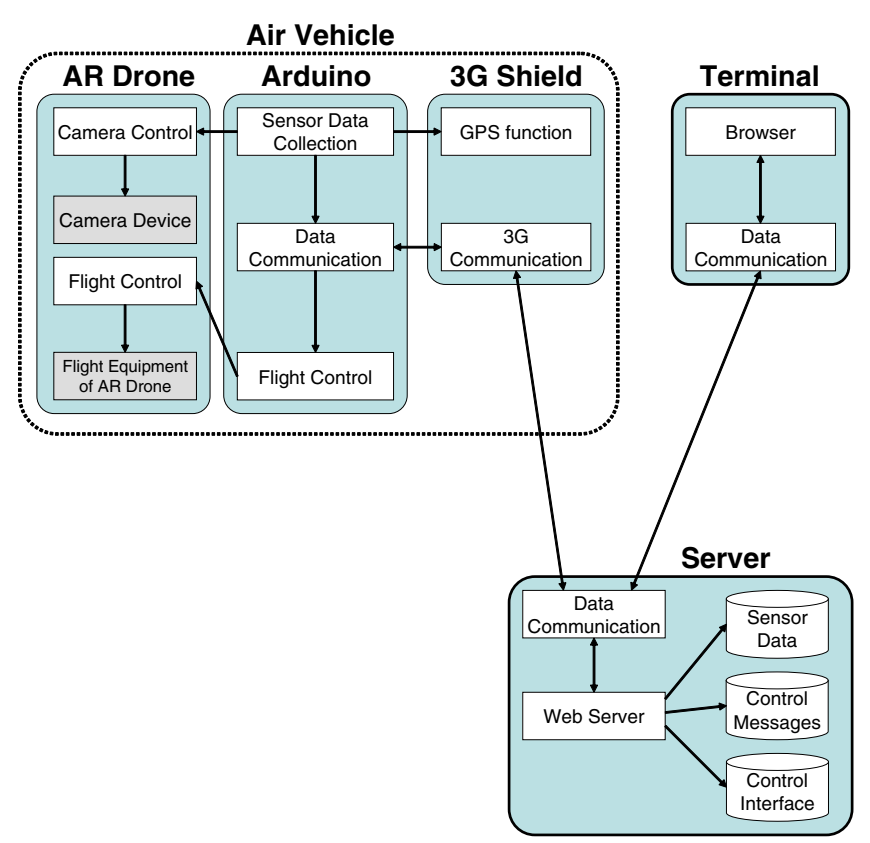

Fig. 4. System Structure of Remote Control System.

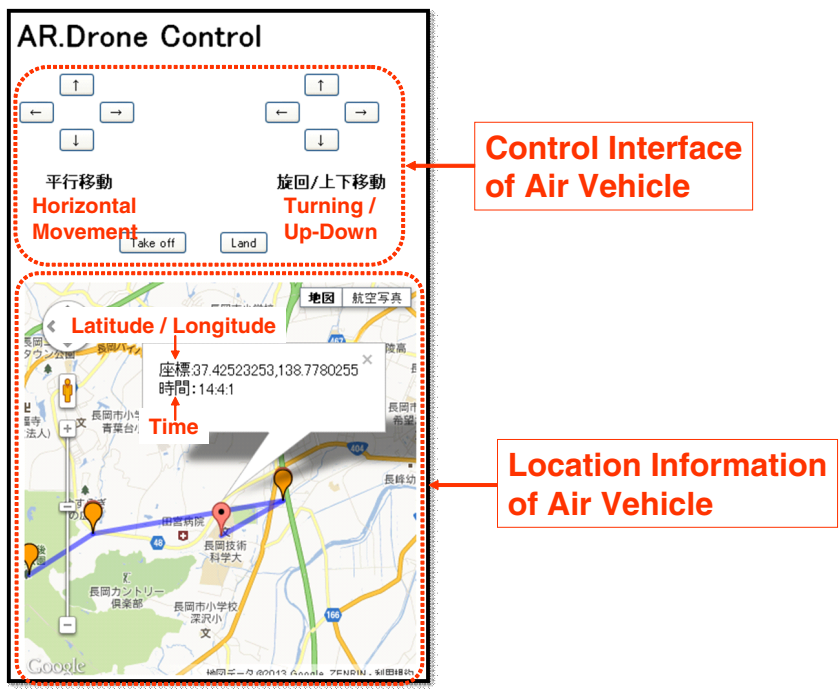

Fig. 5. Control Interface of Air Vehicle.

Figure 4 shows system structure of our proposed remote control system. As shown in this figure, the Arduino works as a central part of the system, and has three main functions. First, "Sensor Data Collection" function collects various sensor data from sensing devices attached to the Arduino. In the latest version of the system, location of the air vehicle can be obtained from the $3 \mathrm{G}$ shield. The function will be updated so as to receive other observation data (e.g., camera image) in the future. Next, "Data Communication" function uploads various observation data to the server, and then receives control messages as the response. Here, the control messages are submitted through the web page (Fig. 5) published by the server, hence the operator can utilize any type of terminal (e.g., laptop 


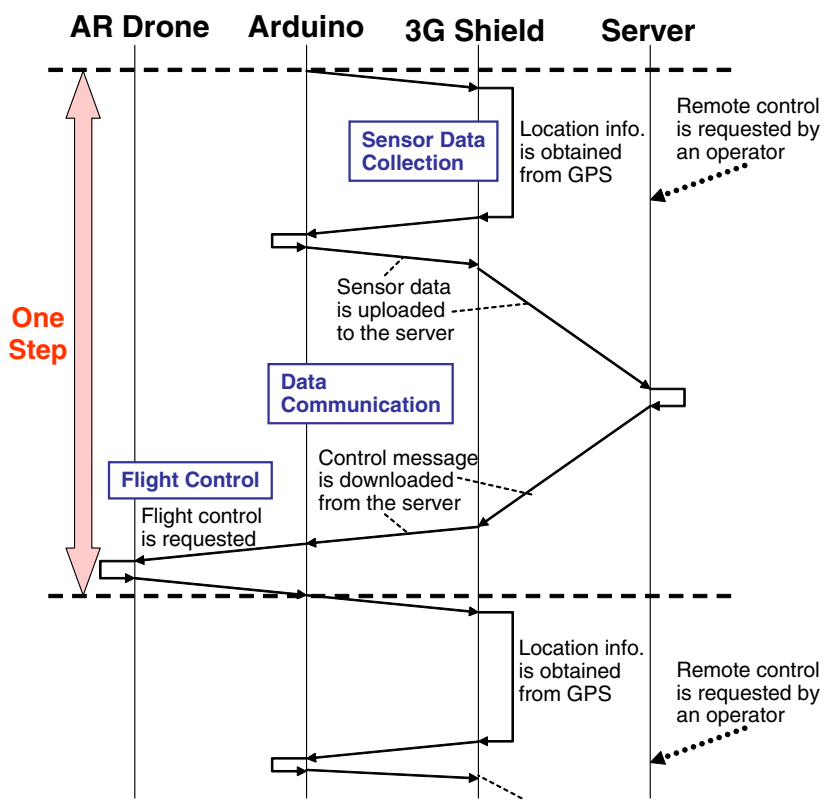

Fig. 6. Sequence Chart of Remote Control System.

PC, tablet, smartphone) which can run a browser application to request flight control of the air vehicle. In addition, as shown in Fig. 5, the web page provides the location of the air vehicle via Google Map to help the operator to control that. The data communication between the air vehicle and the server can be done through the $3 \mathrm{G}$ cellular connection established the by $3 \mathrm{G}$ shield. Third, "Flight Control" function transmits the operater's control request to the AR Drone throught the serial interface. Due to these functions, the operator can control observation process of the air vehicle from everywhere through the $3 \mathrm{G}$ cellular network.

Finally, Figure 6 illustrates a sequence chart of the proposed remote control system. As shown in this figure, the sequence is invoked by the Arduino, and the three functions are sequentially executed, because the CPU (ATmega328p) of the Arduino does not support multi-threading. As illustrated in the sequence chart, network latency between the air vehicle and the server, and response time of receiving location information from GPS are dominant in the control delay. Therefore, these two metrics should be minimized in order to achieve real-time control of the air vehicle in the disaster area.

\section{Experimental Evaluation of Proposed Remote CONTROL SYSTEM}

In order to evaluate feasibility of our proposed remote control system, we performed experimental evaluation in the Nagaoka University of Technology campus.

\section{A. Evaluation Setup}

The air vehicle needs to be controlled through $3 \mathrm{G}$ cellular network in real-time to avoid accidents in the observation field such as collision with building. Therefore, we clarify real-time property of the proposed remote control system by

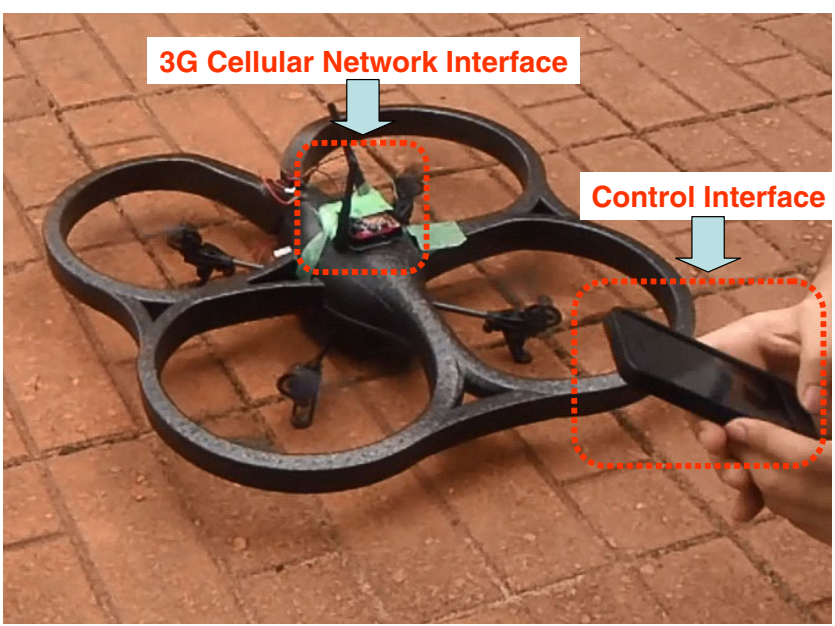

Fig. 7. Setup of Air Vehicle and Control Interface.

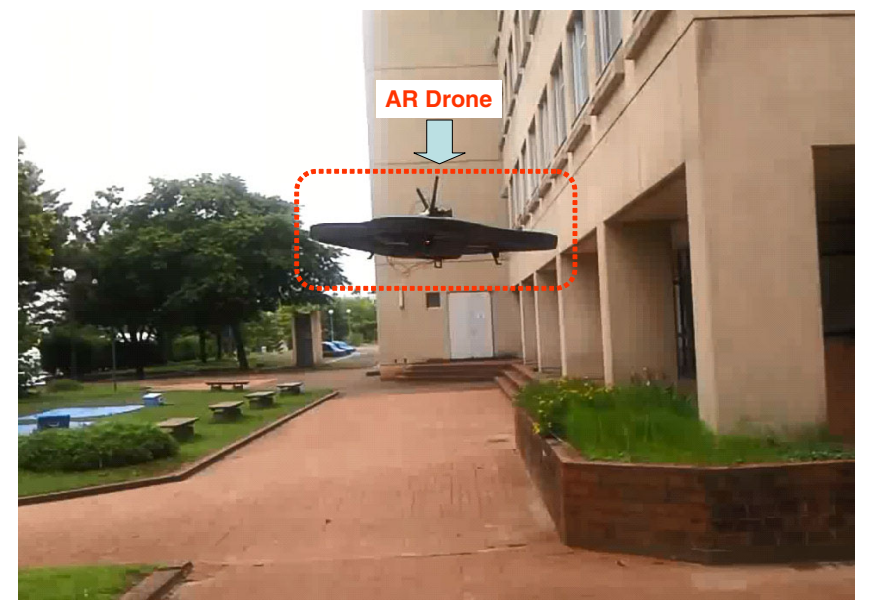

Fig. 8. Flight Test of Air Vehicle.

evaluating control delay needed for the air vehicle to receive control messages from the user terminal. In this evaluation, network latency between the air vehicle and the server and response time of receiving location information from GPS are evaluated, because the increase in these metrics affects the performance as shown in Fig. 6. Here, the Arduino does not have a real-time clock, but is equipped with an API which returns a time interval since it is started. By using the API, the network latency and the response time of the GPS were measured on the Arduino.

Furthermore, the server is deployed on the campus of the Nagaoka University of Technology, and the web page is published by Apache 2.2.9. In the evaluation, an iPhone 5 is used as the user terminal as shown in Fig. 7, and accesses the server through $3 \mathrm{G}$ cellular network to browse the control interface of the air vehicle. As shown in Fig. 8, the AR Drone can flight stably and be accurately controlled through the control interface. Note that any type of terminal (e.g., Android, Windows/Linux PC) can be used to control the air vehicle because the web page does not include any special function depending on the terminal type. 


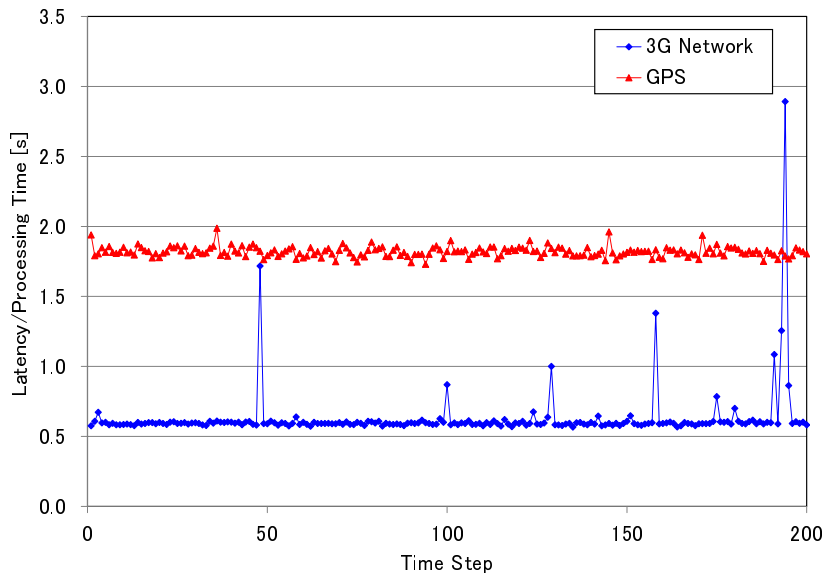

Fig. 9. Processing Time of Remote Control.

\section{B. Evaluation Result}

For evaluating the performance of the proposed remote control system, the network latency on the $3 \mathrm{G}$ cellular network and the response time of GPS are measured two hundreds times. Figure 9 illustrates the measurement results of these metrics in each time step. As shown in this figure, the response time of the GPS is comparably stable, but the network latency sometimes markedly increases due to congestion on the $3 \mathrm{G}$ cellular network.

The average network latency and the average response time were 0.63 and 1.81 [sec], hence the total control delay became more than 2.44 [sec]. The control request by the operator should be applied to the air vehicle in less than one second in order to achieve real-time control of the air vehicle in the disaster area, hence the control delay should be reduced. In the future study, a control program on the Arduino will be updated by adopting new functions (e.g., pseudo multi-threading) so that the response time of the GPS does not affect the total control delay.

\section{CONCLUSIONS}

In this paper, we have proposed and developed a new DTN-based sensor network for disaster observation using air vehicles. Particularly emphasized new technique is a remote control system that enable the operator to control the air vehicles from everywhere so as to obtain detailed observation data from the dangerous field. The remote control system can be achieved by attaching an embedded system, Arduino, with a $3 \mathrm{G}$ cellular module to a commercially-available air vehicle, AR Drone. In addition, the centralized server was prepared on the Internet for building the system where the control messages from the user terminal can be transmitted to the air vehicle by traversing NAT devices. Through the experimental evaluation of the proposed system, it has been clarified that the air vehicle can be controlled through $3 \mathrm{G}$ cellular network, but the control delay was more than $2.44[\mathrm{sec}]$.

Therefore, in the future study, the proposed functions will be improved so that the delay of the remote control can be shorter than one second in order to control the air vehicle in the disaster area in real time. Furthermore, we will consider the database synchronization method between multiple sensor nodes (including the air vehicle itself) and the mail server through aerial data carry by the air vehicles.

This study was partly supported by JSPS KAKENHI Grant Number 25730055.

\section{REFERENCES}

[1] S. M. George, W. Zhou, H. Chenji, M. G. Won, Y. O. Lee, A. Pazarloglou, and R. Stoleru, "DistressNet: A Wireless Ad Hoc and Sensor Network Architecture for Situation Management in Disaster Response," IEEE Communications Magazine, vol.48, no.3, pp.128-136, March 2010.

[2] A. Kamra, V. Misra, J. Feldman, and D. Rubenstein, "Growth Codes: Maximizing Sensor Network Data Persistence," Proc. the 2006 Conference on Applications, Technologies, Architectures, and Protocols for Computer Communications (ACM SIGCOMM 2006), pp.255-266, 2006.

[3] D. Malan, T. Fulford-Jones, M. Welsh, and S. Moulton, "CodeBlue: An Ad Hoc Sensor Network Infrastructure for Emergency Medical Care," Proc. International Workshop on Wearable and Implantable Body Sensor Networks, 2004.

[4] K. Lorincz, D. Malan, T. Fulford-Jones, and A. Nawoj, "Sensor Networks for Emergency Response: Challenges and Opportunities," IEEE Pervasive Computing, vol.3, no.4, pp.16-23, Oct.-Dec. 2004.

[5] K. Fall, "A Delay-Tolerant Network Architecture for Challenged Internets," Proc. the 2003 Conference on Applications, Technologies, Architectures, and Protocols for Computer Communications (ACM SIGCOMM 2003), pp.27-34, 2003.

[6] S. Jain, "Routing in a Delay Tolerant Network," Proc. the 2004 Conference on Applications, Technologies, Architectures, and Protocols for Computer Communications (ACM SIGCOMM 2004), pp.145-158, 2004.

[7] W. Zhao, M. Ammar, and E. Zegura, "A Message Ferrying Approach for Data Delivery in Sparse Mobile Ad Hoc Networks," Proc. the 5th ACM International Symposium on Mobile Ad Hoc Networking and Computing, pp.187-198, 2004.

[8] W. Zhao, and M. Ammar, "Message Ferrying: Proactive Routing in Highly-partitioned Wireless Ad Hoc Networks," Proc. the Ninth IEEE Workshop on Future Trends of Distributed Computing Systems (FTDCS2003), pp.308-314, 2003.

[9] H. Ochiai, H. Ishizuka, Y. Kawakami, and H. Esaki, "A DTN-Based Sensor Data Gathering for Agricultural Applications," IEEE Sensor Journal, vol.11, no.11, pp.2861-2868, 2011.

[10] S. Yamamuraa, A. Nagata, M. Tsuru, and H. Tamura, "Virtual Segment: Store-Carry-Forward Relay-based Support for Wide-Area Non-Real-Time Data Exchange," Simulation Modelling Practice and Theory, Elsevier, vol.19, issue 1, pp.30-46, 2011.

[11] P. Tran Thi Ha, H. Yamamoto, and K. Yamazaki, "Using Autonomous Air Vehicle in DTN Sensor Network for Environmental Observation," Proc. the 37th IEEE Annual International Computers, Software and Applications Conference (COMPSAC2013), pp.447-450, 2013.

[12] AR Drone 2.0, Parrot SA., http://ardrone2.parrot.com/

[13] DJI Phantom, DJI, http://www.dji.com/product/phantom/

[14] Arduino, Arduino, http://www.arduino.cc/

[15] 3G Shield Alliance, TABrain Inc., http://3gsa.org/ 
Hiroshi Yamamoto received M.E. and D.E. degrees from Kyushu Institute of Technology, Iizuka, Japan in '03 and '06, respectively. From April '06 to March '10, he worked at FUJITSU LABORATORIES LTD., Kawasaki, Japan. Since April '10, he has been an Assistant Professor in the Department of Electrical Engineering, Nagaoka University of Technology. His research interests include computer networks, distributed applications, and networked services. He is a member of the IEEE.

Takuya Fujii is currently an undergraduate school student in Nagaoka University of Technology. His research interests include sensor networks and Delay Tolerant Network.

Phuong Tran Thi Ha received B.E. degree from Nagaoka University of Technology in '12. She is currently a graduate school student in Nagaoka University of Technology. Her research interests include sensor networks and Delay Tolerant Network.

Katsuyuki Yamazaki received B.E. and D.E degrees from the University of Electro-communications and Kyushu Institute of Technology in '80 and '01, respectively. At KDD Co. Ltd., he had been engaged in R\&D and international standardization of ISDN, S.S. No.7, ATM networks, L2 networks, IP networks, mobile and ubiquitous networks, etc., and was responsible for R\&D strategy of KDDI R\&D Labs. He is currently a Professor of Nagaoka University of Technology. 